PROCEEDINGS OF THE

AMERICAN MATHEMATICAL SOCIETY

Volume 131, Number 8, Pages 2443-2451

S 0002-9939(02)06801-6

Article electronically published on November 13, 2002

\title{
ISOLATING SEGMENTS FOR CARATHÉODORY SYSTEMS AND EXISTENCE OF PERIODIC SOLUTIONS
}

\author{
MACIEJ J. CAPIŃSKI AND KLAUDIUSZ WÓJCIK \\ (Communicated by Carmen C. Chicone)
}

\begin{abstract}
The method of isolating segments is introduced in the context of Carathéodory systems. We define isolating segments and extend the results of Srzednicki (1994) to Carathéodory systems.
\end{abstract}

\section{INTRODUCTION}

In [S] Roman Srzednicki introduced a geometric method for detecting periodic solutions in nonautonomous periodic differential equations based on the notion of isolating segments. In all practical applications the segments are manifolds with corners contained in the extended phase space, such that at any point on the boundary of the segment the vector field is transversal to the boundary, except the points of external tangency.

In this paper we extend the method of Srzednicki to differential equations, with Carathéodory right-hand sides. We obtain a periodic solution of a Carathéodory system as a limit of periodic solutions to a system of ODE approximating considered Carathéodory system. First we prove that one can choose the approximating sequence of DE's in such a way that their isolating segments (see def below) coincide with the isolating segment of the initial system. By Srzednicki's result [S] this will imply the existence of periodic solutions to approximating equations. From the sequence of these periodic solutions we can choose a subsequence which converges uniformly to a periodic solution for the Carathéodory system.

As an application we consider the work of Andres in $\mathrm{A}$ and next of Górniewicz and Andres in [G] (see also [AGJ]) presenting a nontrivial example of the application of the Nielsen fixed point theory to the investigation of existence and multiplicity of periodic solutions to Carathéodory systems. The problem was suggested by Leray at the International Congress of Mathematicians in 1950. Andres' approach is based on an appropriate functional setting together with the Nielsen theory for a compact map on a metric ANR. Unfortunately there seems to be a gap in the proof presented by Andres. Moreover it seems that Theorem 2 in [A] can be proved using simple geometric arguments.

Received by the editors December 11, 2001 and, in revised form, March 13, 2002.

2000 Mathematics Subject Classification. Primary 34A26, 34B15.

Key words and phrases. Carathéodory systems, periodic solutions, isolating segments.

The second author was partially supported by Polish KBN grant 2 P 03A 02817. 


\section{Preliminaries}

$R^{n}$ is a Euclidean space with a $\cdot$ scalar product and a \|\| norm. $\Delta \subset R^{n}$, $I=[0, T] . \nabla F$ denotes the gradient of $F$. Let us consider a Carathéodory system

$$
\dot{x}=F(t, x)
$$

where $F: \mathbb{R} \times \mathbb{R}^{n} \rightarrow \mathbb{R}^{n}$ is a $T$ periodic function. Put $\Omega=\mathbb{R} \times \mathbb{R}^{n}$. $F$ is Carathéodory in $\Omega$ if

(1) $F(t, \cdot)$ is continuous for a.a. $t$,

(2) the function $F(\cdot, x)$ is measurable for all $x$, and $\|F(\cdot, x)\| \leq \phi(t)$, for all $x$, $\phi$ integrable.

Let us introduce the definition of an isolating segment for Carathéodory systems. Assume that

$$
V_{1}, \ldots, V_{k}: \mathbb{R}^{n} \rightarrow \mathbb{R}
$$

are $C^{1}$ functions. Let $0 \leq r \leq k$. We define a pair of subsets $\left(W_{0}, W_{0}^{-}\right) \subset \mathbb{R}^{n}$ by

$$
\begin{gathered}
W_{0}=\left\{x \in \mathbb{R}^{n}: \forall i \in\{1, \ldots, k\} \mathbb{V}_{i}(x) \leq 0\right\}, \\
W_{0}^{-}=\left\{x \in W_{0}: \exists 1 \leq i \leq r V_{i}(x)=0\right\} .
\end{gathered}
$$

Let

$$
W=[0, T] \times W_{0}, W^{-}=[0, T] \times W_{0}^{-} .
$$

Definition 1. We call $W$ a periodic isolating segment over $I$ and $W^{-}$the exit set of $W$ if

$$
F(t, x) \cdot \nabla V_{i}(x)>0
$$

for all $1 \leq i \leq r$ a.e. in $I$ and $x \in W_{0}$, such that $V_{i}(x)=0$.

$$
F(t, x) \cdot \nabla V_{i}(x)<0
$$

for all $r+1 \leq i \leq k$ a.e. in $I$ and $x \in W_{0}$, such that $V_{i}(x)=0$.

(S3) $W_{0}, W_{0}^{-}$are compact ENR's.

If $F$ is continuous in $t$, then we assume that the inequalities (32) and (4) hold for all $t \in[0, T]$.

The theorem below generalizes Theorem 7.1 in $[\mathrm{S}]$, to the case equations without uniqueness.

Theorem 1. Assume that $F$ is continuous and $W$ is a periodic isolating segment over $[0, T]$ for $F$. If the Euler-Poincaré characteristic $\chi\left(W_{0}, W_{0}^{-}\right) \neq 0$, then there exists an $x \in W_{0}$ such that there is a solution of (i) through $x, T$ periodic and contained in $W$.

Proof. Assume first that $F$ is locally Lipschitzian with respect to $x$. Let $P$ be a Poincaré map (i.e. a map after time $T$ ) associated to $F$. It was proved by Srzednicki that $F_{W}$ (the set of fixed points of the Poincaré map $P$ whose trajectories are contained in the segment $W$ for all times) is open and compact in the set of fixed points of the Poincare map, and the fixed point index $\operatorname{ind}\left(P, F_{W}\right)$ is equal to $\chi\left(W_{0}, W_{0}^{-}\right)$; therefore the theorem is true for functions locally Lipschitzian with respect to $x$. 
Let us now consider the continuous case

$$
x^{\prime}=F(x, t)
$$

where $W$ is an isolating segment for $F$. Since $W$ is compact, there exists a sequence $\left(F_{n}\right)_{n=1}^{+\infty}$ of locally Lipschitzian functions with respect to $x$, uniformly converging to $F$. Since the inequalities (3) and (4) are strict, there exists an $N>0$ such that for all $n>N$ the set $W$ is an isolating segment for $F_{n}$. For all $n>N$ there exists an $x_{n}$ in $W_{0}$ such that the trajectory of the solution of

$$
x^{\prime}=F_{n}(x, t)
$$

starting from $x_{n}$ is $T$ periodic and contained in $W$. Since $W_{0}$ is compact there exists $x_{0} \in W_{0}$, and a subsequence of $\left(x_{n}\right)$ converging to $x_{0}$. The trajectory of the solution of

$$
x^{\prime}=F(x, t)
$$

starting from $x_{0}$ is $T$ periodic and contained in $W$.

The following theorem will later allow us to approximate the solution for a $\mathrm{Ca}$ rathéodory system with the solutions of ordinary differential equations.

Theorem $2([\underline{\mathrm{H}}, \underline{\mathrm{N}}])$. Let $\left\{f_{\nu}\right\}$ be Carathéodory and let

$$
\lim _{\nu \rightarrow \infty} \int_{I} \sup _{x \in \Delta}\left|f_{\nu}(t, x)-f(t, x)\right| d t=0
$$

for every compact rectangle $I \times \Delta \subseteq \Omega$. Moreover, let $\left\{P_{\nu}\right\}, P_{\nu}=\left(t_{\nu}, \xi_{\nu}\right)$ be a sequence in $\Omega$ with $\lim P_{\nu}=P_{0}=\left(t_{0}, \xi_{0}\right)$. Suppose that for any $\nu \in N$, the function $x_{\nu}$ is a solution of the initial value problem

$$
\dot{x}=f_{\nu}(t, x), \quad x\left(t_{\nu}\right)=\xi_{\nu} .
$$

Then there exists a solution $x$ of the problem

$$
\dot{x}=f(t, x), \quad x\left(t_{0}\right)=\xi_{0},
$$

and a subsequence $\left\{x_{k_{\nu}}\right\}$ of $\left\{x_{\nu}\right\}$ such that

$$
\lim x_{k_{\nu}}=x,
$$

uniformly on compact subintervals of Dom $x$.

\section{MAin RESUlT}

Theorem 3. Let $F \in \operatorname{Car}_{l_{\mathrm{loc}}}(\Omega)$ be $T$ periodic and bounded on compact subsets of $\Omega$. If $W$ is an isolating segment for $F$ over $[0, T]$ and $\chi\left(W_{0}, W_{0}^{-}\right) \neq 0$, then there exists an $x \in W_{0}$ such that its trajectory is $T$ periodic and is contained in $W$.

The basic idea of the proof of Theorem 3 is to combine Theorems 1 and 2 . In order to do that we need the functions in Theorem[2 to be continuous. The following lemma will allow us to have that.

Lemma 4. Let $F \in \operatorname{Car}_{\text {loc }}(\Omega)$, be $T$ periodic. Assume that $F$ is bounded on compact subsets of $\Omega$. Put $I=[0, T]$. Then for any compact rectangle $I \times \Delta$ and any $\varepsilon>0$ there exists a continuous function $F_{0}: I \times \Delta \rightarrow \mathbb{R}^{n}$ such that

$$
\int_{I} \sup _{x \in \Delta}\left|F_{0}(t, x)-F(t, x)\right| d t \leq \varepsilon .
$$

Furthermore, if $W \subset I \times \Delta$ is a periodic isolating segment over $I$ for $F$, then $W$ is an isolating segment for $F_{0}$. 
Proof. Let $I \times \Delta$ be fixed. There exists $C>0$ such that $\left|F_{|I \times \Delta|}\right|<C$. Note that $C(\Delta)$ with the supremum norm is a separable Banach space.

Let us define $\Phi(t):=F(t, \cdot), t \in I$. The function $\Phi: I \rightarrow C(\Delta)$ is measurable.

By Luzin's Theorem $[\mathbf{F}$, for any $\varepsilon>0$ there exists a compact set $K$ such that $\mu(I \backslash K) \leq \varepsilon$ and $\Phi_{\mid K}$ is continuous.

There exists a set $B \subset I$ such that $\mu(B)=\mu(I)$ and for any $a \in B$ the inequalities (3) and (4) hold.

For any $a \in K$ there exists an open neighborhood $U_{a}$ such that

$$
\|\Phi(t)-\Phi(a)\| \leq \varepsilon
$$

for any $t \in K \cap U_{a}$.

For some $a_{0} \in(I \backslash K) \cap B$ we put $U_{a_{0}}=I \backslash K$.

Since $B$ is of a full measure in $[0, T], U_{a_{j}} \cap B \neq \emptyset$ for all $j \in\{1, \ldots, N\}$. Hence we can choose a finite covering $U_{a_{1}}, \ldots, U_{a_{N}}$ of $K$ such that

$$
I \subset U_{a_{0}} \cup U_{a_{1}} \cup \ldots \cup U_{a_{m}},
$$

$$
\forall j \in\{0,1, \ldots, N\}, a_{j} \in B .
$$

Let us take a partition of unity

$$
\phi_{j} \in C_{0}\left(U_{a_{j}},[0,1]\right), j=0,1, \ldots, N,
$$

$$
\phi_{0}+\phi_{1}+\ldots+\phi_{N}=1
$$

on $I$. Now we can define

$$
F_{0}(t, x):=\sum_{j=0}^{N} \phi_{j}(t) F\left(a_{j}, x\right) \text { for }(t, x) \in I \times \Delta .
$$

For any $t \in K$ we have $t \notin \operatorname{supp} \phi_{0} \subset I \backslash K$, so

$$
\left\|F_{0}(t, \cdot)-\Phi(t)\right\|=\left\|\sum_{j=1}^{N} \phi_{j}(t) \Phi\left(a_{j}\right)-\sum_{j=1}^{N} \phi_{j}(t) \Phi(t)\right\| \leq \sum_{j=1}^{N} \phi_{j}(t)\left\|\Phi\left(a_{j}\right)-\Phi(t)\right\| \leq \varepsilon .
$$

We know that $\|F(t, \cdot)\| \leq C, t \in I$, so

$$
\begin{aligned}
\int_{I} \sup _{x \in \Delta}\left|F_{0}(t, x)-F(t, x)\right| d t= & \int_{I}\left\|F_{0}(t, \cdot)-F(t, \cdot)\right\| d t \\
= & \int_{I \backslash K}\left\|F_{0}(t, \cdot)-F(t, \cdot)\right\| d t \\
& \quad+\int_{K}\left\|F_{0}(t, \cdot)-F(t, \cdot)\right\| d t \\
\leq & \mu(I \backslash K) 2 C+\mu(K) \varepsilon \\
\leq & (2 C+\mu(K)) \varepsilon .
\end{aligned}
$$

We prove that if $W \subset I \times \Delta$, then the conditions (S1) and (S2) hold for $F_{0}$ with any $t \in I$. Let $(t, x) \in W, i_{0} \in\{1, \ldots, r\}$ (resp. $i_{0} \in\{r+1, \ldots, k\}$ ), be such that $V_{i_{0}}(x)=0$. Then by (3) (resp. (44)), (5) and (6)

$$
F_{0}(t, x) \cdot \nabla V_{i_{0}}(x)=\sum_{j=0}^{N} \phi_{j}(t) F\left(a_{j}, x\right) \cdot \nabla V_{i_{0}}(x)>0(\text { resp. }<0) .
$$


Proof of Theorem [3] Let us choose $I$ and $\Delta$ such that $W \subset I \times \Delta$. From Lemma 4 we know that there exists a sequence of continuous functions $F_{n}$ such that

$$
\int_{I} \sup _{x \in \Delta}\left|F_{n}(t, x)-F(t, x)\right| d t \leq \frac{1}{n}
$$

and $W$ is an isolating segment for all $F_{n}$. By Theorem 1 we know that for every $n$ there exists a point $x_{n} \in W_{0}$ such that the trajectory $\phi_{n}$ generated by $F_{n}$, starting from $x_{n}$, is periodic and contained in $W$. Since $W_{0}$ is compact, there exists a subsequence $\left\{x_{\nu_{n}}\right\}$ of $\left\{x_{n}\right\}$ and a point $x_{0} \in W$ such that $\lim x_{\nu_{n}}=x_{0}$. From Theorem 2 we know that there exists a trajectory $\phi$ which is a solution of the problem

$$
\dot{x}=F(t, x), \quad x(0)=x_{0},
$$

and a subsequence $\left\{\phi_{\nu_{k_{n}}}\right\}$ of $\left\{\phi_{\nu_{n}}\right\}$ such that

$$
\lim \phi_{\nu_{k_{n}}}=\phi
$$

uniformly on $I$.

\section{EXAmple of APPLiCATiOnS}

Consider the planar nonautonomous system $(*)$ of the form

$$
\begin{aligned}
x^{\prime} & =-a x+e(t, x, y) y^{\frac{1}{m}}+g(t, x, y)=F_{1}(t, x, y), \\
y^{\prime} & =-b y+f(t, x, y) x^{\frac{1}{n}}+h(t, x, y)=F_{2}(t, x, y),
\end{aligned}
$$

where $e, f, g, h: \mathbb{R} \times \mathbb{R}^{2} \rightarrow \mathbb{R}$ are Car $_{\text {loc }}\left(\mathbb{R} \times \mathbb{R}^{2}\right)$ functions, $T$ periodic in $t(T>0)$. Moreover $F=\left(F_{1}, F_{2}\right)$ is bounded on the compact subsets of $\mathbb{R} \times \mathbb{R}^{2}$.

The system $(*)$ was presented by Jan Andres in $[\mathrm{A}]$ and next by Górniewicz and Andres in $[\mathrm{AG}]$ as a nontrivial example of an application of the Nielsen fixed point theory.

Theorem 5 ([A] $)$. Assume that

(1) the functions $g, h$ are bounded by positive constants $G$ and $H$, respectively,

(2) there are positive constants $e_{0}, f_{0}, \delta_{1}, \delta_{2}$ such that

$$
0<e_{0}<e(t, x, y),
$$

for a.a. $t \in[0, T]$ such that $(t, x, y) \in\left(\mathbb{R} \times\left\{\delta_{1}\right\} \times\left[\delta_{2},+\infty\right)\right) \cup\left(\mathbb{R} \times\left\{-\delta_{1}\right\} \times\right.$ $\left.\left(-\infty,-\delta_{2}\right]\right)$ and

$$
0<f_{0}<f(t, x, y),
$$

for a.a. $t$ such that $(t, x, y) \in\left(\mathbb{R} \times\left[\delta_{1},+\infty\right) \times\left\{\delta_{2}\right\}\right) \cup\left(\mathbb{R} \times\left(-\infty,-\delta_{1}\right] \times\right.$ $\left.\left\{-\delta_{2}\right\}\right)$

(3) $a b>0$,

(4) $m, n$ are odd integers with $\min (m, n) \geq 3$,

(5)

$$
\begin{aligned}
& \frac{1}{|a|}\left(e_{0} \delta_{2}^{\frac{1}{m}}-G\right)>\delta_{1}, \\
& \frac{1}{|b|}\left(f_{0} \delta_{1}^{\frac{1}{n}}-H\right)>\delta_{2} .
\end{aligned}
$$


(6) there are positive constants $r, M, \alpha, \beta$ such that $\alpha+\frac{1}{m}<1, \beta+\frac{1}{n}<1$ and

$$
|e(t, x, y)| \leq M|x|^{\alpha}
$$

for $|x| \geq r$ and a.a. $t \in[0, T], y \in \mathbb{R}$,

$$
|f(t, x, y)| \leq M|y|^{\beta}
$$

for $|y| \geq r$ and a.a. $t \in[0, T], x \in \mathbb{R}$.

Then the system $(*)$ admits at least three $T$ periodic solutions.

Remark 6. One can check that the sharp inequalities (10) considered by Andres in the periodic case imply (5). Moreover, instead of the boundedness assumption applied in [A] we use a more general assumption (6).

Proof of Theorem [5. Let $F=\left(F_{1}, F_{2}\right)$. We assume that $a, b$ are positive. Replacing $t$ by $-t$ it is clear that the same result also holds for $(*)$ with negative constants.

During the course of the proof we will prove several inequalities, which will later allow us to define appropriate isolating segments, from which we will obtain the result.

Lemma 7. Let $R>0$ be sufficiently large. Then for all a.a. $t \in[0, T]$

(I) $F_{1}(t, R, y)<0$, if $|y| \leq R$,

(II) $F_{1}(t,-R, y)>0$, if $|y| \leq R$,

(III) $F_{2}(t, x, R)<0$, if $|x| \leq R$,

(IV) $F_{2}(t, x,-R)>0$, if $|x| \leq R$.

Proof. (I) $|y| \leq R$ and $t \in[0, T]$, so

$$
\begin{aligned}
F_{1}(t, R, y) & \leq-a R+|e(t, R, y)| R^{\frac{1}{m}}+G \\
& \leq-a R+M R^{\alpha+\frac{1}{m}}+G<0
\end{aligned}
$$

provided $R$ is large enough.

(II) For $|y| \leq R$ and $t \in[0, T]$ we have

$$
\begin{aligned}
F_{1}(t,-R, y) & \geq a R-|e(t,-R, y)| R^{\frac{1}{m}}-G \\
& \geq a R-M R^{\alpha+\frac{1}{m}}-G>0
\end{aligned}
$$

provided $R$ is large enough.

(III) For $y=R,|x| \leq R$ and $t \in[0, T]$ we have

$$
\begin{aligned}
F_{2}(t, x, R) & \leq-b R+|f(t, x, y)| R^{\frac{1}{n}}+H \\
& \leq-b R+M R^{\beta+\frac{1}{n}}+H<0
\end{aligned}
$$

provided $R$ is large enough.

(IV) For $y=-R,|x| \leq R$ and $t \in[0, T]$ we have

$$
\begin{aligned}
F_{2}(t, x,-R) & \geq b R-|f(t, x,-R)| R^{\frac{1}{n}}-H \\
& \geq b R-M R^{\beta+\frac{1}{n}}-H>0
\end{aligned}
$$

provided $R$ is large enough.

Lemma 8. For a.a. $t \in[0, T]$ the following conditions hold:

(A) $F_{1}\left(t, \delta_{1}, y\right)>0$ for $y \geq \delta_{2}$,

(B) $F_{2}\left(t, x, \delta_{2}\right)>0$ for $x \geq \delta_{1}$, 
(C) $F_{1}(t,-\delta, y)<0$ for $y \leq-\delta_{2}$,

(D) $F_{2}\left(t, x,-\delta_{2}\right)<0$ for $x \leq-\delta_{1}$.

Proof. By (1), (5)

$$
F_{1}\left(t, \delta_{1}, y\right) \geq-a \delta_{1}+e_{0} \delta_{2}^{\frac{1}{m}}-G>0
$$

for $y \geq \delta_{2}$,

$$
F_{2}\left(t, x, \delta_{2}\right) \geq-b \delta_{2}+f_{0} \delta_{1}^{\frac{1}{n}}-H>0
$$

for $x \geq \delta_{1}$,

$$
F_{1}\left(t,-\delta_{1}, y\right) \leq a \delta_{1}-e_{0} \delta_{2}^{\frac{1}{m}}+G>0
$$

for $y \leq-\delta_{2}$,

$$
F_{2}\left(t, x,-\delta_{2}\right) \leq b \delta_{2}-f_{0} \delta_{1}^{\frac{1}{n}}+H<0
$$

for $x \leq-\delta_{1}$.

Let $W=[0, T] \times[-R, R] \times[-R, R]$. By Lemmas 7 and 8 , the sets

$$
\begin{gathered}
W=[0, T] \times[-R, R] \times[-R, R], \\
V=[0, T] \times\left[\delta_{1}, R\right] \times\left[\delta_{2}, R\right], \\
U=[0, T] \times\left[-\delta_{1},-R\right] \times\left[-\delta_{2},-R\right], \\
Z=\operatorname{cl}(W \backslash(U \cup V)),
\end{gathered}
$$

are the periodic isolating segments for $F$ over $[0, T]$. Since $U^{-}=V^{-}=\emptyset$ and

$$
\chi\left(U_{0}, U_{0}^{-}\right)=\chi\left(V_{0}, V_{0}^{-}\right)=1,
$$

by Theorem 3 there are two $T$ periodic solutions, one contained in $U$ and one in $V$. One can check that $Z_{0}^{-}$has two components:

$$
[0, T] \times\left[\delta_{1}, R\right] \times\left\{\delta_{2}\right\} \cup[0, T] \times\left\{\delta_{1}\right\} \times\left[\delta_{2}, R\right]
$$

and

$$
[0, T] \times\left[-R,-\delta_{1}\right] \times\left\{\delta_{2}\right\} \cup[0, T] \times\left\{-\delta_{1}\right\} \times\left[-R,-\delta_{2}\right] .
$$

Since $\chi\left(Z_{0}, Z_{0}^{-}\right)=-1$, again by Theorem 3 the third $T$ periodic solution is contained in $Z$.

Remark 9. It follows by the proof that the existence of two periodic solutions (contained in the segments $U$ and $V$ ) can also be deduced by the Brouwer fixed point theorem.

In the work of Andres and Górniewicz [AG the example is also considered.

Remark 10 ([AG]). On the basis of the Schauder fixed point theorem one can get only one $T$ periodic solution of $(*)$. If, additionally, condition (2) holds for a.a. $t \in[0, T]$ and all $(x, y) \in R^{2}$, then at least three $T$ periodic solutions of $(*)$ can also be deduced by means of the fixed-point index technique or at least two $T$ periodic solutions, when applying the Schauder fixed-point theorem. 


\section{REMARK ON THE APPROACH BY NIELSEN FIXED POINT THEORY}

In $[\mathrm{A}]$, a different approach to the problem is considered. Condition (2) is more restrictive:

$(2)^{\prime}$ For $x \geq-\delta_{1}, y \geq \delta_{2}$ and a.a. $t$ as well as for $x \leq \delta_{1}, y \leq-\delta_{2}$ and a.a. $t$

$$
0<e_{0}<e(t, x, y) .
$$

For $x \geq \delta_{1}, y \leq \delta_{2}$ and a.a. $t$ as well as for $x \leq-\delta_{1}, y \geq-\delta_{2}$ and a.a. $t$

$$
0<f_{0}<f(t, x, y)
$$

The problem is approached by the application of the following theorem.

Theorem 11. Let $G: J \times \mathbb{R}^{n} \times \mathbb{R}^{n} \rightarrow \mathbb{R}^{n}$ be a Carathéodory mapping, where $J$ is an arbitrary interval. Assume, furthermore, that there exists a (nonempty) closed connected subset $Q$ of $C\left(J, \mathbb{R}^{n}\right)$ such that the problem

$$
X^{\prime}=G(t, X, q(t)), \quad X \in S,
$$

has, for every $q \in Q$, a unique solution $X(t)=T(q)$ with the property $\operatorname{cl}(T(Q)) \subset S$, where $S$ is a (nonempty) bounded subset of $C\left(J, \mathbb{R}^{n}\right)$, and $T: Q \rightarrow S$ is retractable onto $Q$.

At last there exists a locally Lebesgue-integrable function $\alpha: J \rightarrow \mathbb{R}$ such that

$$
|G(t, X(t), q(t))| \leq \alpha(t) \text { a.e. in } J
$$

for any pair $(q, X) \in \Gamma_{T}$, where $\Gamma_{T}$ denotes the graph of $T$.

Then the Carathéodory system

$$
X^{\prime}=F(t, X)
$$

admits at least $N\left(\left.r\right|_{T(Q)} \circ T().\right)$ solutions belonging to $Q$, provided $G(t, c, c)=F(t, c)$ takes place a.e. in $J$, for any $c \in \mathbb{R}^{n}$.

As the constraint $S$, the following set is considered:

$$
S=Q=Q_{1} \cap Q_{2} \cap Q_{3},
$$

where

$$
\begin{gathered}
Q_{1}=\left\{q(t) \in C\left([0, \omega], \mathbb{R}^{2}\right):\|q(t)\|=\max \left[\max _{t \in[0, \omega]}\left|q_{1}(t)\right|\right.\right. \\
\left.\left.\max _{t \in[0, \omega]}\left|q_{2}(t)\right|\right] \leq R\right\} \\
Q_{2}=\left\{q(t) \in C\left([0, \omega], \mathbb{R}^{2}\right): \min _{t \in[0, \omega]}\left|q_{1}(t)\right| \geq \delta_{1}>0\right. \\
\text { or } \left.\min _{t \in[0, \omega]}\left|q_{2}(t)\right| \geq \delta_{2}>0\right\} \\
Q_{3}=\left\{q(t) \in C\left([0, \omega], \mathbb{R}^{2}\right): q(0)=q(\omega)\right\}
\end{gathered}
$$

The Carathéodory mapping takes the form

$$
\begin{aligned}
x^{\prime}+a x & =e\left(t, q_{1}(t), q_{2}(t)\right) q_{2}(t)^{\frac{1}{m}}+g\left(t, q_{1}(t), q_{2}(t)\right), \\
y^{\prime}+b y & =f\left(t, q_{1}(t), q_{2}(t)\right) q_{1}(t)^{\frac{1}{n}}+h\left(t, q_{1}(t), q_{2}(t)\right) .
\end{aligned}
$$

For each $q \in Q$ the problem has a unique solution $T(q)$,

$$
T(q)=\left\{\begin{array}{l}
x(t)=\int_{0}^{\omega} G_{1}(t, s)\left[e\left(s, q_{1}(s), q_{2}(s)\right) q_{2}(s)^{\frac{1}{m}}+g\left(s, q_{1}(s), q_{2}(s)\right)\right] d s \\
y(t)=\int_{0}^{\omega} G_{2}(t, s)\left[f\left(s, q_{1}(s), q_{2}(s)\right) q_{1}(s)^{\frac{1}{n}}+h\left(s, q_{1}(s), q_{2}(s)\right)\right] d s
\end{array}\right.
$$

where

$$
G_{1}(t, s)=\left\{\begin{array}{c}
\frac{e^{-a(t-s+\omega)}}{1-e^{-a \omega}} \text { for } 0 \leq t \leq s \leq \omega \\
\frac{e^{-a(t-s)}}{1-e^{-a \omega}} \text { for } 0 \leq s \leq t \leq \omega
\end{array}\right.
$$




$$
G_{2}(t, s)=\left\{\begin{array}{l}
\frac{e^{-b(t-s+\omega)}}{1-e^{-b \omega}} \text { for } 0 \leq t \leq s \leq \omega, \\
\frac{e^{-b(t-s)}}{1-e^{-b \omega}} \text { for } 0 \leq s \leq t \leq \omega .
\end{array}\right.
$$

In order to apply Theorem 11 it is necessary to check that $\operatorname{cl}(T(Q)) \subset S=Q$.

This condition need not always be satisfied. The problem lies with the condition $T(Q) \subset Q_{2}$. As an example let us choose a problem with $g=h=0$. The solution for $t=0$ is

$$
\begin{aligned}
& x(0)=\int_{0}^{\omega} \frac{e^{-a(\omega-s)}}{1-e^{-a \omega}} e\left(s, q_{1}(s), q_{2}(s)\right) q_{2}(s)^{\frac{1}{m}} d s, \\
& y(0)=\int_{0}^{\omega} \frac{e^{-b(\omega-s)}}{1-e^{-b \omega}} f\left(s, q_{1}(s), q_{2}(s)\right) q_{1}(s)^{\frac{1}{n}} d s .
\end{aligned}
$$

It is possible to choose $\left(q_{1}, q_{2}\right) \in Q$ and bounded $e, f$ satisfying condition $(2)^{\prime}$, such that $x(0)=y(0)=0$, hence $T\left(\left(q_{1}, q_{2}\right)\right) \notin Q_{2}$.

\section{ACKNOWLEDGEMENTS}

The authors would like to thank Professor Marek Jarnicki and Professor Stanisław Sędziwy for their helpful discussions.

\section{REFERENCES}

[A] J. Andres "A nontrivial example of application of the Nielsen fixed-point theory to differential systems: problem of Jean Leray" Proc. AMS (2000) vol. 128, 2921-2931. MR 2000m: 47074

[AGJ] J. Andres, L. Górniewicz, J. Jezierski "Noncompact version of the multivalued Nielsen theory and its application to differential inclusions" Differential Inclusions and Optimal Control, Lecture Notes in Nonlinear Analysis, vol. 2, (1998), 33-50.

[AG] J. Andres, L. Górniewicz "From the Schauder Fixed-Point Theorem to The Applied Multivalued Nielsen Theory" TMNA vol. 14, (1999), 229-238. MR 2001h:47092

[F] H. Federer "Geometric measure theory". Die Grundlehren der mathematischen Wissenschaften, Band 153 Springer-Verlag New York Inc., New York, 1969. MR 41:1976

[G] A. Granas "The Leray-Schauder index and the fixed point theory for arbitrary ANR's" Bull. Soc. Math. France 100 (1972), 209-228. MR 46:8213

[H] P. Hartman "Ordinary Differential Equations" John Wiley, New York, 1964. MR 30:1270

$[\mathrm{N}] \quad$ S. Nakagiri, H. Murakami "Kneser's property of solutions families of nonlinear Volterra integral equations" Proc. Japan Acad. 50 (1974), 296-300. MR 50:14138

[S] R. Srzednicki "Periodic and bounded solutions in blocks for time-periodic nonautonomous ODE's" Nonlinear Anal. TMA 22 (1994), 707-737. MR 95c:34076

Institute of Mathematics, Jagiellonian University, Reymonta 4, 30-059 Kraków, Poland

E-mail address: mcapinsk@im.uj.edu.pl

Institute of Mathematics, Jagiellonian University, Reymonta 4, 30-059 Kraków, Poland

E-mail address: wojcik@im.uj.edu.pl 